\title{
Block design of a wheelset for railway transport
}

\author{
Alexander Shiler ${ }^{1, *}$, Valeriy Shiler ${ }^{1}$ and Ilkham Galiev ${ }^{1}$ \\ ${ }^{1}$ Omsk State Transport University, Karla Marks Ave., 35, 644010, Omsk, Russia
}

\begin{abstract}
The paper presents an analysis of the features of geometric contacting of the rolling surfaces of a block design of a wheelset and a rail. The peculiarities of the contact slipping by the rolling surfaces of the contact stresses are considered.
\end{abstract}

\section{Introduction}

As is known, the increase in the volume of rail traffic can be mastered in two ways: by increasing the size of traffic and the weight of trains. The first method is mainly reduced to an increase in the traffic capacity of existing lines, as well as the construction of new ones. A retrospective statistical analysis shows $[1,2]$ that if the level of traffic capacity usage exceeds rational, then the service and technical speeds decrease sharply, and this, in turn, leads to significant disruptions in the work of the transport conveyor, material and social losses. The second method involves increasing the axial load and weight of trains.

Let's consider the dynamics of the main technical and economic indicators of railway transportation of the Russian Federation over a 50 years period. For example: the average service speed of train ranged from 37 to $40 \mathrm{~km} / \mathrm{h}$, the axial load increased from 21.5 tons to 23 - 25 tons, the weight standard of the train from 4000 to $7000 \div 9000$ tons. With these values, the maximum possible traffic capacity of the double-track section was up to 120 pairs of trains per day, which was the norm at the Omsk-Novosibirsk section during the period under review. Currently, a further increase in carrying capacity is planned by increasing the axial load to 27 tons and 29 tons. Such a way will lead to increased wear of the track superstructure, wheelsets, and rolling stock. For example, from our experience: with the transition from the $1524 \mathrm{~mm}$ gauge to the $1520 \mathrm{~mm}$ gauge (narrowing just $4 \mathrm{~mm}$ ), the intensity of the side wear of the wheel flanges has increased by twenty times! and spread along the entire length of the main tracks of RZD. The introduction of lubrication has reduced wear by six times, but the intensity of the side notch is three times higher than previous indicators. When using electric locomotives with an axle load of 25 tons at the Novokuznetsk-Toshtogol section (95\% of the length of this direction are curved sections of the track), the side notch of wheel flanges was intensively formed (within ten days of operation of these electric locomotives, flanges were worn down to $25 \mathrm{~mm}$ in thickness). It is noteworthy that after a short operation (two - three months) of electric locomotives with an axle load of 25 tons at this section, an increased notch of flanges began to be observed also in other types of rolling stock, which had satisfactory indicators for this type of wear until this moment. Later, the same effect spread to the side surface of the rails. Thus, these

*Corresponding author: shiler_alex@inbox.ru 
examples convincingly show that the system "rolling stock - track" is a single "organism". The changes in the parameters in the design of any rolling stock unit must be coordinated with the parameters of the entire system "rolling stock - track".

\section{Block wheel design}

In the present work, a block design of a wheelset is proposed, which, with an increased axial load, will reduce the resistance to movement and significantly reduce the intensity of side wear of the flanges and rails. This effect in the mechanical system "block wheelset - track" is obtained due to the "disclosure" of all three closed contours. In a large family of existing wheelsets, this technical solution is implemented for the first time.

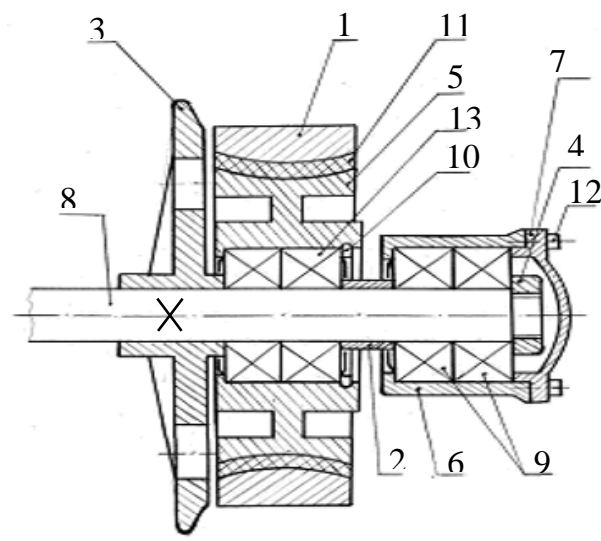

Fig. 1. New design of a wheelset wheel for a rail vehicle.

The block design of a wheel of a wheelset is shown in fig. 1, which consists of a disk 3 and a wheel center 5 rotating independently of each other. The disk 3 is rigidly mounted on the axis 8 , which has the ability to rotate in axle box bearings 9 . The disk 3 has the shape of a tyre flange along the perimeter and perceives horizontal directing forces from the side surface of the rails. The wheel center 5 by means of a pair of bearings 13 is installed on the axis of the wheelset 8 and transmits the vertical load of the weight of the rolling stock to the flexible rim 1, which rolls along the rolling surface of the rail. The space between the wheel center 5 and the tyre 1 is filled with an elastic material 11, in particular rubber.

\section{Analysis of the trajectories of contacts of the block wheel flange}

To determine the nature and parameters of the interaction of the block design of the wheel with the surfaces of the rail head, the analysis of the trajectories of the points of contact of the rolling surface of the tyre (K0) and the flange (B0) was performed. Thus, the trajectory of the point (K0) has the form of an ordinary cycloid (see Fig. 2, line 3). The point of contact of the flange B0 moves along the trajectory of a conventional cycloid (see Fig. 2, line 1), provided that the angle of attack of the flange on the rail is zero. As is known, the main feature of a conventional cycloid is the equality of the distance traveled by the center of the generating circle and its total length, which corresponds to the movement of the wheel without slipping. For comparison, the trajectory of the point of contact of the flange of a standard wheelset, which has the form of an elongated cycloid, is shown (see Fig. 2, line 2). 


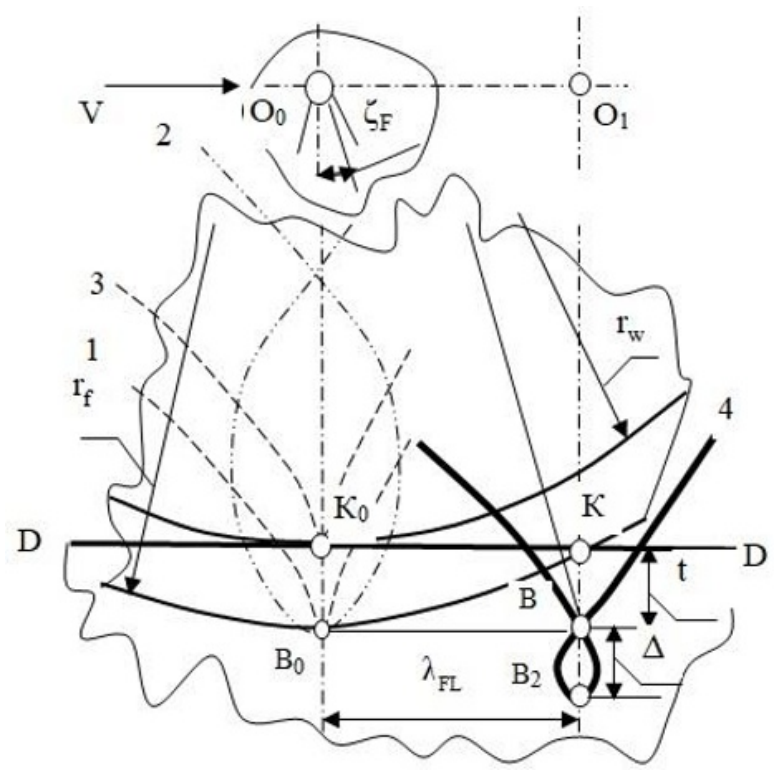

Fig. 2. The trajectory of the point of contact of the wheel flange (B1) along the side surface of the rail head in the presence of an "overrun" $(\lambda \mathrm{FL})$.

The presence of an elongated cycloid loop on the trajectory indicates that the point of the generating circle repeats the already traveled path, and, consequently, the presence of parasitic slippage of the flange along the side surface of the rail head.

In the presence of the angle of attack of the flange on the rail, the point of contact of the flange of the block wheelset (B1) is displaced relative to the vertical axis of the wheel by the value of the "overrun" $(\lambda F L)$. In the presence of the "overrun", the point of contact of the flange (B1) traces out a trajectory that corresponds to the determinant of the curvature curve of the "conchoids of Nicomedes" (see Fig. 2, line 4).

\section{The method of calculating the length of the slip of the flange along the side surface of the rail}

By definition of a conchoids, the point (B1) is a pole, and the straight line $\mathrm{O} 0 \mathrm{O} 1$ is its base. The trajectory of the conchoid was built for the radius O0B1. In accordance with the method of building a conchoid, the point B1 is the instantaneous center of rotation of the flange. In the process of translational motion of the wheel, the segment (O0B1), which is a generator of the flange surface, slides at the point (B1) of the side surface of the rail head in two directions: rotation relative to the point (B1) and linear movement along the radius (OB1). Taking into account the smallness of the contact area at the point (B1) and the width of the trajectory of the conchoid loop, further calculations of the length of the flange slipping path along the rail head do not take into account the rotation at the point B1 of the generator O0B1 (spin-moment). Therefore, in the presence of an "overrun" $(\lambda F L)$, the length of the flange slipping path along the rail head is estimated by the length of the vertical displacement $(\Delta=$ B1B2) (see Fig. 2), which is determined from the geometric relationships in a right triangle OOB0B1:

$$
\Delta=\sqrt{\left(r_{\mathrm{w}}+\mathrm{t}\right)^{2}+\lambda_{\mathrm{FL}}^{2}}-\left(\mathrm{r}_{\mathrm{w}}+\mathrm{t}\right)
$$


where rw - the radius of the rolling surface of the wheel, $\mathrm{mm}$;

$\mathrm{t}$ - the height of the location of the point of contact of the flange (B0) with the side surface of the rail head relative to the rolling surface of the rail (K0), (usually $\mathrm{t}=10 \mathrm{~mm}$ ) $\mathrm{mm}$;

$\lambda \mathrm{FL}$ - the value of the "overrun" of the point of contact of the flange (B1) and the rail head relative to the vertical axis of the wheel (B0K0) is determined by the formula [5]:

$$
\lambda_{\mathrm{FL}}=\left(r_{\mathrm{w}}+\mathrm{t}\right) \cdot \operatorname{tg}(\alpha+\gamma) \cdot \operatorname{tg} \tau
$$

where $\alpha-$ the angle of attack of the wheel on the rail (the maximum possible value is $3^{\circ}$ );

$\tau$ - the angle of inclination of the working surface of the flange to the horizon;

$\gamma$ - the angle of obliquity of the rail track.

In a standard wheelset, the point (B1) makes a rotational motion relative to the instantaneous center of rotation, which is the point K0 (see Fig. 2, line 2). When the flange of the rail head is touched, the rotational movement of the point (B1) forms the trajectory of the elongated cycloid. The instantaneous radius ( $\mathrm{rB} 1)$ of the point (B1) relative to the instantaneous center (K0) is determined by the equation:

$$
r_{\mathrm{B}_{1}}=\sqrt{t^{2}+\lambda_{\mathrm{FL}}^{2}}
$$

The length of the slipping path of the point of contact of the flange (B1) along the rail head for one turn of the wheel has the following dependence:

$$
L_{\mathrm{B}_{1}}=2 \cdot \pi \cdot r_{\mathrm{B}_{1}}=2 \cdot \pi \cdot \sqrt{t^{2}+\hbar_{\mathrm{FL}}^{2}}
$$

\section{Results of calculations of the length of slipping of the flange along the side surface of the rail}

Using equations ( 1 and 4 ), we calculated the lengths of slipping of the flanges along the rail head for standard and new wheel designs for one turn of the wheel, which are shown in the graph (see Fig. 3) for both wheelsets' designs.

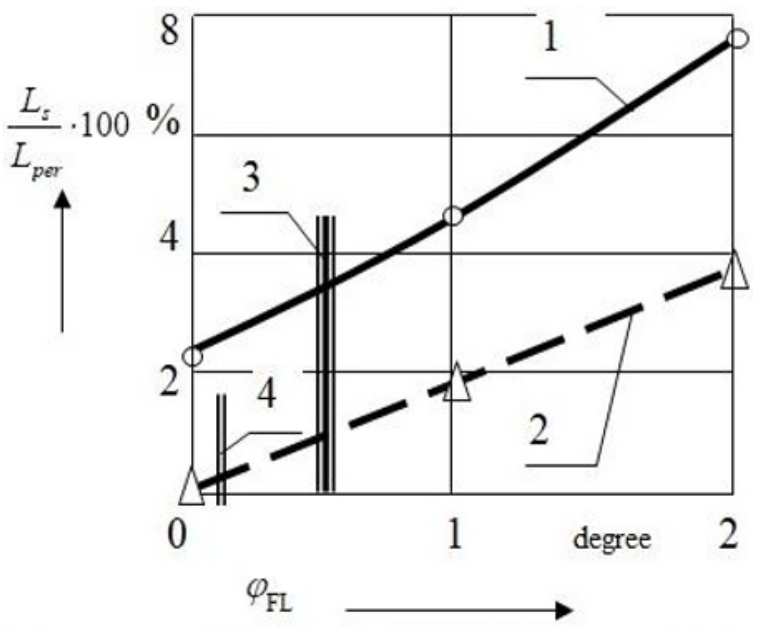

Fig. 3. Dependence of the reduced length of the flange slip along the rail head of the angel of its attack on the rail: 1 - standard wheelset; 2 - new design of a wheelset. The maximum value of the angel of attack of the flange on the rail: 3 - standard wheelset; 4 - new wheel design. 
The main advantage of the new wheel design is that with a zero value of the angle of attack of the flange (see Fig. 3, curve 2), it rolls along the side surface of the rail head without slipping. According to the graph, in the whole interval of values of the angle of attack $(\alpha)$, the length of the slipping path of the flange of the new wheel design is significantly less $(2 \div$ 8 times), compared to the standard wheelset (see Fig. 3, curves 2 and 1, respectively). According to the results of field studies and theoretical calculations, it was found that on the straight part of the path, the maximum angle of attack reaches $0.5^{\circ}$ [2-4] for a standard wheelset, and for a new wheelset design $-0.2^{\circ}$ (see Fig. 3, lines 3 and 4 , respectively).

\section{Profiles of rolling surfaces and the distribution of contact stresses on the elements of the block wheel and rail}

In addition to reducing the "parasitic" slip in the new design of the wheelset, new opportunities have been obtained for reducing the contact stresses in the "wheel-rail" system through the use of a new transverse profile of the rolling surface of a flexible tyre.

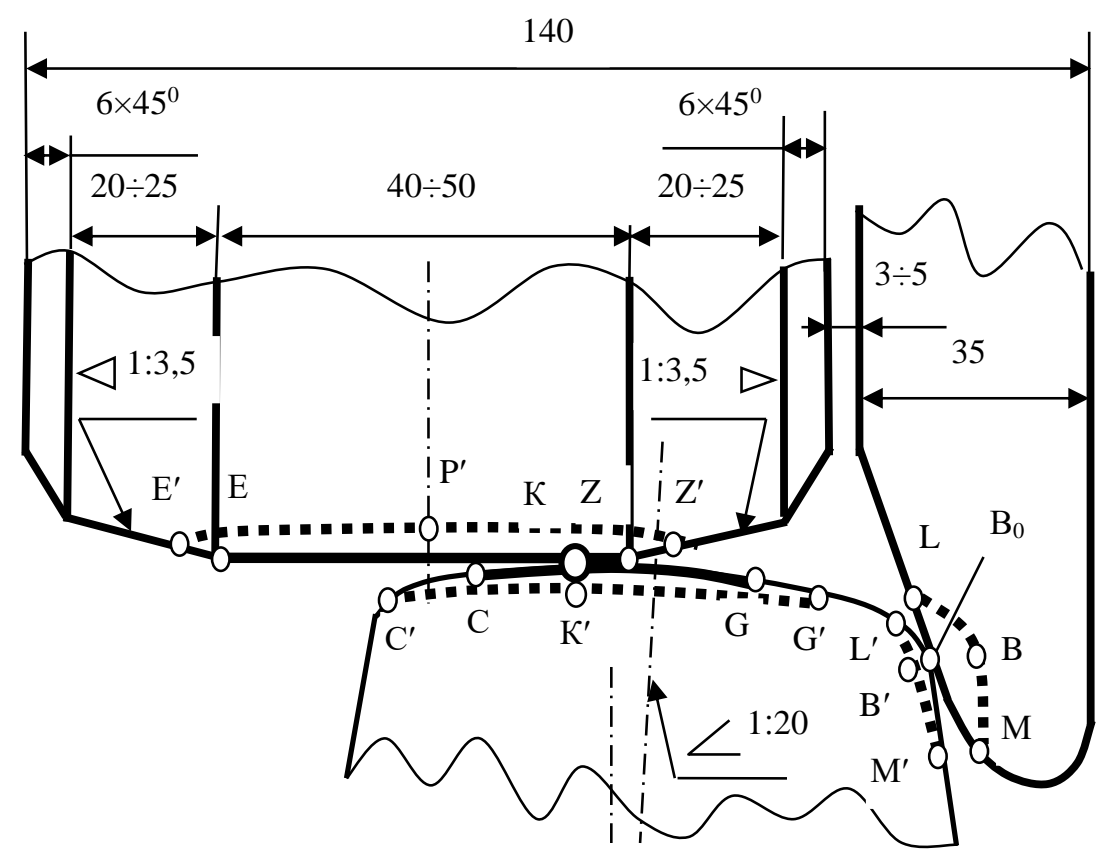

Fig. 4. Transverse profile of the rolling surface of the flexible rim.

So, in the presence of independent rotation of the rolling surfaces of flexible tyres, there is no function to compensate for the difference in the diameters of their rolling circles, and, consequently, the need for a taper of the rolling surface equal to $1: 10$. Due to the independently rotating flexible tyres and the flange disk, the need to fillet the cross section was automatically eliminated. Taking into account the requirements on the profile of a standard wheelset, the new transverse profile of the rolling surface of a flexible tyre $[5,6]$ is formed from the five surfaces shown in Fig. 6 . The central part of the rolling surface of the flexible tyre (see Fig. 4, section EZ) is made in the form of a cylindrical surface. Both edge sections of the transverse rolling profile have the same outlines in the form of two conical surfaces, which are also used in the standard profile: cone $\triangleleft 1: 3,5$ and chamfer $6 \times 45^{\circ}$. To ensure a stable outline of a comfortable profile, the width of the cylindrical part is assumed 
to be $50 \mathrm{~mm}$. This value is obtained from the following relationship: the sum of the lengths of the cylindrical part (EZ) and two five millimeter segments ( $\mathrm{EE}^{\prime}$ and $\mathrm{ZZ}$ ) is equal to the maximum value of the open gap between the wheel and rail track at a curved section of the road that is $40 \mathrm{~mm}$. Such a choice of dimensions ensures the stability of the outline of the comfortable profile of the rolling surface (section EZ) during operation due to the wear of the segments $\left(\mathrm{EE}^{\prime}\right.$ and $\mathrm{ZZ}$ ). The profile of the side surface of the flange has the same configuration as that of the standard wheel flange.

The main advantage of the new flexible tyre profile is the single-point contact of the rolling surface of the flexible tyre (section EZ) in the central part of the rail head surface at any position of the flexible tyre relative to the rail and at any level of wear on the contacting surfaces. Taking into account the rail canting, the contact point $\left(\mathrm{K}^{\prime}\right)$ is located on the outer half of its rolling surface at a distance of $7 \div 10 \mathrm{~mm}$ from the vertical axis of symmetry. When the flange is overrunning on the rail head, two contact points are formed, which are located at a significant distance from each other, which ensures uniform distribution of contact stresses along the entire transverse perimeter of the rail head and the wheel. Due to the independent rotation of the flange of a relatively flexible tyre, there is nearly no "parasitic" slippage at the contact points.

\section{Conclusion}

A block design of a wheelset is proposed, which, when implemented with increased axial loads, significantly reduces the stress state on the rolling surfaces of the elements of the block wheelset and the rail and their mechanical wear.

\section{References}

1. Yu.M. Luzhnov, Adhesion of wheels with rails (nature and laws) (Intext, Moscow, 2003)

2. U.J. Harris, S.M. Zakharov, J. Landgren, Generalization of the best practices of heavy traffic: issues of the interaction of a wheel and rail (Intext, Moscow, 2002)

3. I.A. Pankin, Railway Transport 7, 52 - 56 (1992)

4. M.F. Verigo, A.Ya. Kogan, Interaction of the track and rolling stock (Transport, Moscow, 1986)

5. I.I. Galiev, V.V. Shiler, P.I. Gorbunov, K.A. Kychakov, V.A. Nikolaev, D.V. Talovskiy, Pat. No. 2378127 Russian Federation, IPC H 60 B 9/I2 (2009)

6. G.I. Petrov and A.A Tarmaev, Proc. of the 6th Int. Symposium on Innovation and Sustainability of Modern Railway ISMR 2018. Beijing, China Railway Publishing House, 426-431 (2018) 